\title{
Les émeutes entre hindous et musulmans (partie 2)
}

\section{Christophe Jaffrelot}

\section{(2) OpenEdition}

12 Journals

\section{Édition électronique}

URL : http://journals.openedition.org/conflits/756

DOI : $10.4000 /$ conflits.756

ISSN : $1777-5345$

Éditeur :

CCLS - Centre d'études sur les conflits lilberté et sécurité, L'Harmattan

\section{Édition imprimée}

Date de publication : 21 mai 1992

ISSN : 1157-996X

\section{Référence électronique}

Christophe Jaffrelot, "Les émeutes entre hindous et musulmans (partie 2) », Cultures \& Conflits [En ligne], 05 | printemps 1992, mis en ligne le 02 janvier 2003, consulté le 30 mars 2021. URL : http:// journals.openedition.org/conflits/756 ; DOI : https://doi.org/10.4000/conflits.756

Ce document a été généré automatiquement le 30 mars 2021.

Creative Commons License 


\title{
Les émeutes entre hindous et musulmans (partie 2)
}

\author{
Christophe Jaffrelot
}

La gestion des violences intra-communautaires

1 L'extrême rareté des émeutes au Bengale occidental ${ }^{1}$ (peuplé à $22 \%$ environ de musulmans) où les communistes, au pouvoir depuis 1977, sont réputés pour leur sécularisme rigoureux, constitue un indice de la dimension proprement politique du problème. Dans d'autres régions, en effet, les chefs du Congrès, se montrent parfois intéressés à gérer cette violence de diverses façons. Le cas de figure le plus avéré consiste, pour des hommes exclus du pouvoir, à discréditer le parti ou la faction de leur formation à la tête du gouvernement d'un état fédéré en démontrant, par une émeute à laquelle ils contribuent ou qu'ils provoquent, que le pouvoir régional est incapable de maintenir "la loi et l'ordre". Deux exemples récents illustrent ces pratiques. A Indore, en 1989, l'émeute évoquée plus haut a été orchestrée par les hommes de l'ancien chef du gouvernement du Madhya Pradesh qui avait été remplacé par M. Vora, le leader d'une autre faction du Congrès régional, à la suite d'une affaire de corruption: l'émeute, vraisemblablement provoquée par un homme de main dans un contexte tendu par l'agitation nationaliste hindoue, visait à mettre ce rival en difficulté à l'approche des élections, période où chaque chef de faction cherche à obtenir le maximum d'investitures du parti pour les siens ${ }^{2}$.

2 Un scénario analogue s'est déroulé à Hyderabad en décembre 1990. Cette capitale de l'Andhra Pradesh qui compte $25 \%$ de musulmans fut le cadre d'une émeute de sept jours qui fit officiellement 120 morts (officieusement le double), en grande partie des hindous de la vieille ville où se concentrent, comme souvent, les musulmans. Or, ces violences semblent bien avoir été déclenchées par des provocations dont l'initiative revenait à un groupe congressiste dissident soucieux de faire chuter le chef du gouvernement qui, de fait, démissionna ${ }^{3}$. Il est vrai que l'atmosphère avait été très tendue par une tournée du président du BJP dans la ville et par l'agitation nationaliste hindoue en général. Il est d'ailleurs nécessaire que ces techniques de violences 
calculées se greffent sur l'action préalable de militants de l'une ou l'autre communauté religieuse.

3 Le second cas de figure de cette gestion de la violence impliquant des gouvernants concerne leur recours différencié aux forces de l'ordre. L'analyse des motivations est ici rendue plus difficile parce que les stratégies sont plus subtiles et parfois non couronnées de succès. Notre constat de départ porte sur la lenteur avec laquelle les forces de l'ordre s'emploient parfois à maîtriser les émeutes. Celles-ci tendent de ce fait à se prolonger parfois plus d'une semaine. Ce phénomène s'explique d'abord par des raisons politiques. Les gouvernants régionaux peuvent retarder une intervention efficace des forces de l'ordre pour trois types de raison : ou bien donner satisfaction à la communauté des agresseurs qui peuvent être musulmans, comme à Hyderabad où ce raisonnement semble bien avoir été tenu pendant plusieurs jours par le chef du gouvernement; ou bien laisser l'émeute se développer contre la minorité musulmane pour mieux se présenter ensuite comme son protecteur attitré, un rôle auquel le Congrès prétend depuis longtemps ; ou encore lui "donner une leçon" à l'approche des élections lorsqu'elle a refusé un soutien massif, lors du précédent scrutin, au détenteur du pouvoir.

4 Que ces objectifs soient atteints ou non, leur poursuite soumet les autorités administratives à des interférences incessantes de la part des potentats locaux. Les cadres de la police et des administrations locales y voyaient d'ailleurs déjà en 1981 une cause majeure de leur incapacité à prévenir et réprimer au plus vite les émeutes ${ }^{4}$. Il s'agit là cependant d'une façon commode de se dédouaner, en particulier de la part des polices régionales du nord de l'Inde, comme la Provincial Armed Constabulary (PAC) qui dépend du gouvernement d'Uttar Pradesh et dont le biais antimusulman est notoire. Celui-ci, en partie lié à la faible représentation des musulmans - 300 sur 200 000 - au sein de cette administration ${ }^{5}$, se traduit de plus en plus souvent par une abdication de leur rôle d'arbitres de la part de policiers qui tendent à se joindre, voire à se substituer aux émeutiers hindous ${ }^{6}$.

5 Cette conjonction des pratiques policières et des tactiques politiques s'est observée le plus nettement (en dépit de la marge d'incertitude inhérente à ces collusions secrètes) dans les émeutes de Meerut en 1982 et 1987 où des membres de la PAC ont abattu chaque fois des dizaines de musulmans ${ }^{7}$. En 1982, le président du Congrès de la ville, depuis longtemps frustré par le choix systématique de candidats musulmans - logique dans une agglomération où cette communauté représente $49 \%$ des habitants - pour les élections locales, aurait contribué à l'émeute, notamment en faisant libérer les premiers fauteurs de troubles arrêtés par la police, sans doute pour démontrer sa capacité de nuire aux musulmans ${ }^{8}$. En 1987, le chef du gouvernement d'Uttar Pradesh lui-même semble avoir laissé se développer l'émeute qui n'était apparemment pas sans liens avec la désaffection des musulmans pour les candidats congressistes locaux: des meurtres et des pillages eurent lieu alors même qu'il se trouvait sur place. Il retarda alors le retrait de la PAC et le déploiement de l'armée qui, là comme ailleurs, devenait le seul recours ${ }^{9}$. Les responsables politiques au plus haut niveau favorisent aussi cette dérive en évitant ou en refusant de prendre les sanctions qui s'imposent : en 1987, par exemple, seul le commandant de la PAC servant à Meerut fut suspendu, une mesure dont la légèreté "pourrait créer l'impression que la PAC peut agir en toute impunité", comme le soulignait Amnesty International dans un rapport d'enquête ${ }^{10}$. Cette clémence peut s'expliquer par le caractère inopportun que comporte parfois la moindre 
sanction dans un contexte préélectoral ${ }^{11}$. De façon générale, le recours à des sanctions est compliqué par la dépendance envers la complicité des forces de police dans laquelle se trouvent les politiciens pour accomplir leurs stratégies.

6 Ces deux ressorts politiques de l'émeute ou de sa propagation -l'éviction d'un rival au pouvoir ou le parasitage des actions de police - peuvent aussi se combiner pour donner lieu à un troisième cas de figure : celui où l'émeute vise l'éviction de cadres de la police jugés trop intègres par les politiciens locaux. Il semble que cette motivation - à laquelle se réfère $U$. Chatterjee, en exergue, dans le cadre d'un ouvrage de fiction très réaliste ait constitué un des facteurs de l'émeute de Jamshedpur en 1979. De fait, le nouveau superintendant de police gênait la classe politique locale par sa rigueur et sa droiture ; discrédité par les violences, il fut transféré dans un autre lieu ${ }^{12}$.

7 Les trois volets de cette gestion de la violence intercommunautaire conduisent finalement à analyser ce phénomène en termes d'un déclin de l'état de droit lié à la criminalisation croissante du jeu politique. D'un côté, les interférences avec la logique des forces de l'ordre sont une condition permissive - au minimum - du développement des émeutes, de l'autre, le déclenchement d'émeutes dans le cadre de rivalités pour le pouvoir implique que les politiciens à l'origine du processus disposent d'hommes de main se recrutant dans des bandes hors-la-loi qu'il faut pouvoir protéger des policiers trop zélés. Le plus souvent, il s'agit de contrebandiers pratiquant le trafic de la drogue, des alcools (dans les états où une prohibition est en vigueur comme le Goudjerat), ou diverses exactions couvertes par leur patron politique qu'ils peuvent donc aider, en échange, lors d'émeutes ou de bourrages d'urnes. Ces hommes de main constituent, après ceux chargés des basses „uvres dans la nébuleuse nationaliste hindoue, une seconde catégorie d'acteurs impliqués de façon concrète dans les violences intercommunautaires. Le fait est avéré, parmi les émeutes déjà citées, à Indore et Bhagalpur. Dans ce dernier cas, les violences semblent même avoir été aussitôt relayées par des gangs en cheville avec un candidat local battu aux dernières élections : pour les premiers, c'était un bon moyen de faire transférer pour incompétence le nouveau superintendant de police, plus actif que ses prédécesseurs contre la pègre, pour le second, il s'agissait de "donner une leçon" musulmans qui ne l'avaient pas soutenu lors du dernier scrutin ${ }^{13}$.

Conclusion

8 Le type idéal de l'émeute intercommunautaire des années 1980 semble finalement se situer à l'intersection d'un faisceau de facteurs que l'on s'est efforcé de hiérarchiser. Les différents rituels valorisés par l'approche "culturaliste" constituent une toile de fond nécessaire mais ne fournissent sans doute pas une explication suffisante des violences dans la mesure où ces données ne sont souvent à l'origine des émeutes que sous la forme de prétextes à d'autres types de conflits. Ceux-ci ont pu être d'abord d'ordre socioéconomiques et locaux dans les années 1960-1970, comme l'indique le modèle microsociologique "économiciste", mais les enjeux politiques paraissent plus déterminants depuis le début des années 1980. On observe en effet lors de cette décennie la généralisation d'un modèle ancien, mais jusqu'alors plutôt marginal, qui se décompose en plusieurs éléments. Il s'agit tout d'abord d'une (a) mobilisation nationaliste hindoue de réaction contre le panislamisme de la minorité musulmane qui s'articule autour de (b) processions pseudo-religieuses en forme de démonstrations de force, ces provocations (c) permettant de déclencher des émeutes aux connotations politiques (voire électorales) à un niveau panindien. Ce modèle se trouve cependant 
compliqué par les tentatives de certains politiciens pour utiliser ces violences dans le cadre d'un déclin de l'état de droit.

Cette nouvelle donnée réintroduit une dimension locale dans la mesure où l'ampleur d'une émeute semble désormais conditionnée par le degré de criminalisation du jeu politique en un lieu donné. Cette règle d'analyse reçoit un début de validation avec le cas de Bhagalpur: il semble en effet logique que la plus meurtrière des émeutes récentes ait eu lieu au Bihar, l'état qui, de tous, est en proie à la crise du droit la plus nette. De telle sorte qu'on doit probablement situer l'émeute à l'intersection des facteurs internationaux (impact du panislamisme), nationaux (rôle des nationalistes hindous) et régionaux ou locaux (degré de criminalité politique). Le niveau intermédiaire, celui de la stratégie nationale des militants hindous parait toutefois prépondérant dans la mesure où c'est à cet échelon que les craintes suscitées par les développements au plan international sont articulées de façon idéologique à des fins politiques, tandis que les politiciens régionaux ou locaux d'une autre obédience se contentent de tirer le meilleur parti des tensions et des violences.

L'approche économiciste et microsociologique partage cependant des zones de chevauchement avec le modèle plus idéologique et politique que l'on a essayé d'exposer ici. Les récentes émeutes de Bhagalpur et de Bénarès se sont par exemple aussi traduites par la destruction des métiers à tisser ou des stocks d'artisans musulmans de la soie. Mais les observateurs, significativement, ont remarqué que les auteurs de ces violences visaient moins que par le passé les biens économiques qu'ils ne cherchaient à faire de victimes physiques ${ }^{14}$. Cette évolution reflète de notre point de vue la pénétration de l'idéologie nationaliste hindoue à la faveur d'un contexte international puis intérieur des plus propices au cours des années 1980 ; comme l'explique G. Pandey, "ce qui se trouve derrière cette brutalité folle, incroyable $(\Sigma)$, c'est la conviction que les victimes sont des monstres véritables ou potentiels qui nous auraient fait la même chose, ou même pire, s'ils en avaient eu ne serait-ce que la moitié d'une occasion"15. L'analyse de Pandey ne porte toutefois que sur les foules mises en cuvre, pour partie du moins, par la propagande des nationalistes hindous. Or, ceux-ci répondent à une logique qu'il ne faut pas négliger.

11 Le véritable dénominateur commun de l'approche économiciste microsociologique et de notre modèle réside précisément dans la valorisation des stratégies rationnelles sous-jacentes aux explosions de violence qui semblent a priori spontanées et sans logique. Cette approche conduit à isoler deux principaux types d'acteurs émeutiers, les hommes de main des mouvements nationalistes hindous et ceux des patrons politiques. Cette catégorisation ne doit toutefois pas occulter le rôle des polices à l'origine d'un grand nombre des victimes, soit en situation de répression, soit en situation d'agression.

La dimension rationnelle des émeutes n'en exclut cependant pas toute référence culturelle; au contraire, elle implique qu'on utilise des institutions culturelles mobilisatrices comme les processions hindoues. Mesurer l'ampleur de la réinterprétation de ces pratiques religieuses et leur importance dans les émeutes entre hindous et musulmans par rapport à d'autres types de violence requiert sans doute ici un effort de comparaison. Le contexte indien offre en effet à l'analyse d'autre exemples de ce recours à "l'émeute comme action rationnelle "à travers les violences intercastes et en particulier celles affectant des milieux intouchables. Certaines de ces émeutes 
s'avèrent d'ailleurs amenées par des "rituels de provocation" incorporant l'usage de processions"16.

Il convient, pour finir, de relativiser l'importance d'un phénomène que notre effort d'analyse, ne serait-ce qu'en l'isolant, a peut-être grossi : les émeutes entre hindous et musulmans, demeurent un fait sporadique et/ou localisé. Moins que l'ampleur de ces violences, c'est la tendance qui ne laisse pas d'inquiéter. Or, le gouvernement formé en 1991 paraît acquis à une vigoureuse politique de réhabilitation de l'état de droit laïc. Les discours de Narasimha Rao, dont les accents sécularistes rappellent le style de Nehru, ont connu un début de concrétisation à travers le vote d'une loi gelant le statut des lieux de culte et l'annonce d'une force de police anti-émeutes ${ }^{17}$. Cette prise de conscience de la gravité de la situation semble s'être étendue à certain Etats fédérés : le gouvernement du Bihar lui-même s'est attaché à indemniser et à reloger les victimes musulmanes de l'émeute de Bhagalpur ${ }^{18}$.La réalité de ces changements demandera à être testée dans l'épreuve car l'accalmie constatée depuis les derniers mois de 1991 doit beaucoup au souci des nationalistes hindous de maintenir la loi et l'ordre - un critère d'appréciation majeur de l'électorat - dans les Etats dont ils ont formé le gouvernement en 1990 (Himachal Predesh, Rajasthan, Madhya Predesh) et en 1991 (Uttar Pradesh ). Or cette préoccupation peut disparaître ou se trouver subordonnée à de nouvelles considérations si la relance du "mouvement d'Ayodhya" apparaissait par exemple comme le thème porteur d'une nouvelle campagne électorale.

Abréviations

BJP : Bharatiya Janata Party (Parti du peuple indien)

RSS : Rashtriya Swayamsevak Sangh (Association des volontaires nationaux)

VHP : Vishva Hindu Parishad (Association hindou universelle)

\section{NOTES}

1. Exception faite de villes comme Murshidabad (théâtre de violences en 1988) où la tension entre communautés est alimentée par l'immigration de bangladeshis.

2. Dainik Bhaskar, 21 / 10 / 1989. Des luttes de factions analogues au sein du Congrès local auraient Eté à l'origine de l'émeute de Surat (Goudjerat) à l'approche des élections à l'Assemblée de l'Etat Indian Express, 02/03/1990, p. 5).

3. The Sunday Observer, 16.12.1990 ; et AA. Engineer, "Making of the Hiderabad riots", Economic and Political Weekly 09/02/1991, pp. 271-274. Les violences se sont propagées à certaines zones rurales d'Anrlhra Pradesh quand des militants du BJP y ont ramené le corps des migrants hindous - généralement de jeunes travailleurs du bâtiment - dont les quartiers étaient au centre de l'émeute d'Hyderabad.

4. H. A. Gani, Problems of minority, op. cit. p. 18. Un éditorial publié au moment des Ram Shila Puja mettait d'ailleurs en cause les responsables du Congrès au plus haut niveau : "L'inquiétude des chefs de la nation par rapport aux conséquences politiques qu'entraînerait une attitude de fermeté contre la Vishva Hindu Parishad (dont la campagne était fort populaire) est si nette qu'aucun superintendant de police de 
district ne sait comment traiter la situation intra-communautaire, parce que personne ne peut dire qu'elle sert la préférence de ses maîtres politiques à la fin de la journée", (H. Khare, "Sparring with Communalism - Why Sadhus (renonçants hindous) are setting National Agenda", Times of India, 20. 10. 1989, p. 6.

5. S. 5abenua1 and M. Hasan, "Communal riot in Morardabad", op. cit., p. 4.

6. Le premier cas observé aurait ici été celui de l'émeute de Bénarès en 1977 (A. Banerjee, "Comparative curfew" : changing dimensions of communal politics in India : in V Das ed., Mirrors of violence, op. cit., p. 56.

7. On aurai retrouvé 68 corps à Maliann, ù proximité de Meerut. (A. A Engineer (ed.), Delhi-Meerut riots, New Delhi, Ajanta, 1988, pp. 258-259).

8. A.A. Engineer, "The guilty men of Meerut", Economic and Political Weekly, 6/ 11 / 1982 , p. 1804.

9. AA. Engineer, "Meerut, shame of the nation", AA. Engineer (ed.), Delhi-Meerut riots, op. cit., p. 22.

10. A.G. Noorani, "Amnesty reports on Meerut hillings", Economic and Political Weekly 12/12/1987 p. 2140

11. A Bhagalpur, par exemple, Rajiu Gandhi, à la veille du scrutin, annulera l'ordre de transfert d'un superintendant de police qui avait manifesté un biais fortement antimusulman mais jouissait d'une réelle popularité parmi la majorité hindoue (voir le commentaire acerbe d'un ancien responsable de la police : N.S. Saksena, Communal riots in India, Naida, Trishsul, 1990, p. 20)

12. Cette ville présentait alors bien des conditions propices à l'éclatement de violences intra-communautaires puisqu'on y observait aussi une concurrence économique entre des petits industriels musulmans et hindous réfugiés du Pendjab. M.J. Akbar Riot after riot, Delhi, Penguin books, 1988, pp. 20-22.

13. India Week, 3/11/1989,p. 2.

14. I. Bharti, "Bhagalpur riots and Bihar government", Economic and Political Weekly, 2/12/1989, p. 2 ; et Times of India, 18/11/1991.

15. G. Pandey, "In defence of The fragment", op. cit., p. 566.

16. Voir notamment O. M. Lynch, "Rioting as a rational action. An interpretation of the April 1978 riots in Agra", Economic and Political weekly, 28/11/1981, pp. 1951-1956, où le point de départ de l'offensive de membres de hautes castes contre des intouchables en voie d'ascension sociale et politique fut une procession véhiculant l'image du leader intouchable, Ambedhar, à l'occasion de son anniversaire.

17. Times of India, 27/11/1991.

18. India Today 15/03/1992, pp. 34-35.

\section{RÉSUMÉS}

Si le caractère très ancien et les facteurs religieux des émeutes entre hindous et musulmans ont $\mathrm{pu}$ accréditer une interprétation culturaliste du phénomène, cette analyse s'est rapidement trouvée relayée par une approche économiciste et microsociologique mettant l'accent sur les rivalités socio-économiques locales comme ressort des violences. Cette lecture, sans doute 
pertinente dans les années 1960-1970, ne permet guère de rendre compte des émeutes de la dernière décennie qui s'expliquent d'abord dans une perspective politique. Le " complexe d'infériorité majoritaire " des hindous face à une communauté musulmane perçue comme adossée à une internationale islamique et favorisée par le pouvoir a en effet été exploitée par des groupes nationalistes hindous, notamment dans de] contextes préélectoraux : l'institution hindoue que constitue la procession s'est par exemple trouvée politisée et transformée en démonstration de force à l'origine de nombreuses émeutes. Par ailleurs, les pouvoirs locaux ou régionaux ont davantage tendu à gérer ces tensions au mieux de leurs intérêts, surtout dans les zones où l'Etat de droit était déjà en déclin. Les violences des dernières années suggèrent donc un modèle politico-idéologique où l'émeute se situe au carrefour de facteurs internationaux (l'impact du " panislamisme ") nationaux (la propagande nationaliste hindoue) et locaux (le degré de criminalisation de la politique).

Riots between Hindus and Muslims, due to their ancient origin, have long been explained on religious grounds. Later, economic and micro-sociological explanations were adopted, stressing local socio-economic conflicts. While these interpretations may have remained valid during the sixties and seventies, they failed in the last decade during which riots appear to be based more on political reasons. There exists a " majority inferiority complex " of the Hindu population which perceives the Muslim population as benefiting from international Islamic back-up and governmental preference ; this feeling is exploited by Hinduist groups, particularly for electoral purposes. For instance, the traditional role of the Hindu procession has been perverted into a demonstration of force, often at the origin of riots. Moreover, local and regional authorities especially there where the rule of Law is on the decline - have exploited such tensions for their own benefit. Violence in recent years thus suggests an ideological model at the crossroads of international factors (the " Pan Islamic " impact), national factors (Hindu nationalist propaganda) and local factors (criminalisation of political life).

INDEX

Index géographique : Inde

Mots-clés : émeutes, identité, mobilisation, religion, culture(s)

\section{AUTEUR}

\section{CHRISTOPHE JAFFRELOT}

Chargé de recherches au CERI-CNRS 